\title{
NON-WASTE AND RESOURCE-SAVING RADIATION PROCESS OF POLYMER MODIFIED WOOD PRODUCTION ${ }^{\#}$
}

\author{
A.Avilov, V.Deruyga, G.Popov ${ }^{*}$, V.Rudychev, I.Zalyubovsky \\ Kharkiv State University, Kharkiv, Ukraine
}

\begin{abstract}
Technology of modification wood with synthetic polymer is designed. It is useful for improvement of consumer value of low-grade soft wood such as poplar, aspen, alder or birch as well as for utilization of wood-making industry wastes such as cuttings, sawdust and chips. Composite materials are manufactured by impregnation of wood with synthetic monomers or oligomers and subsequent radiation polymerization. The materials excel all kinds of natural wood in their resistance to abrasion and corrosion. The development was carried out using a linear electron accelerator with electron energy $5-8 \mathrm{MeV}$ and beam power $5 \mathrm{~kW}$.
\end{abstract}

\section{INTRODUCTION}

The development of radiation technology of various polymer composite materials has been held at Kharkiv State University for the last few years [1-6]. Such base components as wood, fiber-board, paper; gypsum, tuff, calcite, sand and their mixtures, wastes of paper, textile and wood; crumbs and dust of marble, granite, gypsum, asbestos-cement, concrete, ceramic are used as the materials base. These capillary-porous materials are impregnated with liquid binder (synthetic monomer or oligomer). For polymerization of the binder in bulk of the materials we use microwave, electron or gamma radiation with energy less than $10 \mathrm{MeV}$.

It is found that by varying the conditions of polymerization one can significantly change the properties of the final material and obtain variety of products from the same row supplies. Low-value raw materials and waste products can be utilized with low energy consumption. When using molding, casting or extrusion for shaping goods one can have an extremely low waste of products. Polymer modified wood (PMW) having attractive look and exceptional durability is the first among developed materials to enjoy commercial success.

\section{TECHNOLOGICAL PROCESS}

The process of radiation-chemical modification of wood for production of chipboard, plywood veneer or another goods includes the following operations: drying of wooden supplies, bunching, degassing, impregnation with

\footnotetext{
"Work was supported by the STCU, Kyiv, Grant \#155

*Email: popov@pht.univer.kharkov.ua
}

synthetic monomer or oligomer, hermetic encapsulation/shaping, radiation treatment, unloading of the end product, trimming/abrasion. The technological scheme of the radiation-chemical process of polymermodified wood formation is shown in Fig.1.

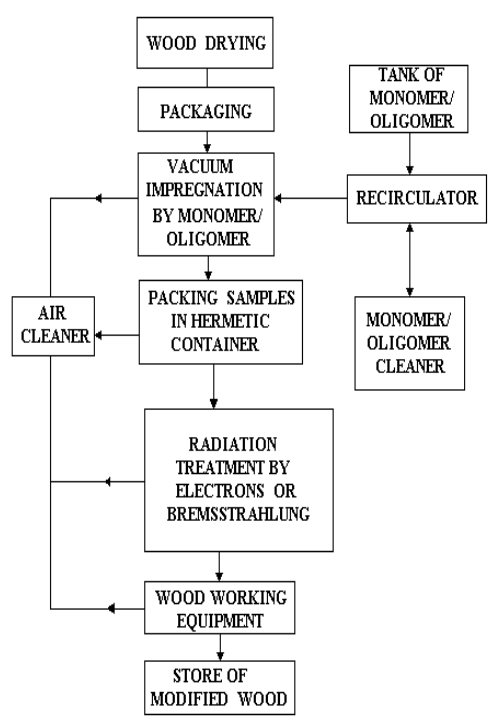

Fig.1. The technological scheme of polymer-modified wood formation under action of relativistic electrons or bremsstrahlung radiation.

The drying is carried out in microwave chambers or vacuum-thermal drying boxes. Dry wood or wooden articles are packed up in metal cassettes which measure $100 \times 10 \times 70 \mathrm{~cm}^{3}$. Each cassette can hold up to 150 parquet strips or correspondent number of solid blocks of material (usually $100 \times 10 \times 7 \mathrm{~cm}^{3}$ ). Our $1 \mathrm{~m}^{3}$ vacuum chamber can hold up to 16 cassettes. Impregnation of wood with binder is carried out by "vacuum - atmospheric pressure overpressure" method. After pumping the chamber it is filled with liquid binder from recirculating reservoir. Treatment time depends on necessary impregnation degree (40 -100 mass per cent).

After impregnation each cassette with wood is put to a light, transparent for radiation frame hermetically wrapped with $0.3-0.5 \mathrm{~mm}$ aluminum foil. These containers are placed consequently on a conveyor delivering them into the radiation field formed by scanning electron beam. Each container may pass the 
sides are irradiated.

\section{EQUIPMENT}

The facilities for PMW formation incorporate a microwave chamber for drying of wooden supplies, a vacuum system for evacuating of oxygen and residual moisture from base material, the system for impregnation of evacuated samples with liquid synthetic binder, facilities for purification of monomer/oligomer and air in work area, radiation-chemical installation for binder polymerization, woodworking equipment for machining of natural and modified wood and equipment for testing of physical and mechanical properties of PMW. The radiation-chemical installation includes a linear electron accelerator, the system for the electron beam scanning, a bremsstrahlung converter, the system for electron beam parameters control and radiation monitoring, and the technology control system. The linear electron accelerator "Electronica - U003" is used as a radiation source. It has the following characteristics: electron energy 4-8 MeV; electron beam current $0.5 \mathrm{~mA}$; mean beam power $5 \mathrm{~kW}$; pulse duration 1-4 $\mu \mathrm{s}$; pulse frequency $1-250 \mathrm{~Hz}$; frequency of electromagnetic scanner 1-8 Hz; the deviation angle of the scanner $\pm 20^{\circ}$. The electron energy is converted to bremsstrahlung radiation with a tantalum converter placed in front of the container.

Beam parameter control system [7-9] is based on original $\mathrm{rod} /$ plate shaped thermoacoustic detectors. It allows measuring of the following instantaneous or integral parameters of electron/bremsstrahlung beam: intensity, diameter and position on the target, effective width, particle distribution in the beam cross section and on the surface of target, cumulative dose of radiation. It makes it easy to adjust radiation beam for particular technology needs.

\section{EXPERIMENTAL RESULTS}

The process of radiation-induced composite material formation for several combinations of base and binder was studied. Such kinds of wood as aspen, alder, poplar, birch, beech, oak, hornbeam and yew ware taken as the base. Vinylacetate, methylmethacrylat, butilmethacrylat, styrene, polyesther- and epoxy resins and their mixtures were used as the binder. Wide and flat samples (around $100 \times 50 \mathrm{~cm}^{2}$ ) were treated by scanning electron or bremsstrahlung beams. Plane distribution of radiation flux on the surface of large samples was achieved by using a special form modulation current to feed the electromagnet of the scanning system. The samples, more than $100 \mathrm{~cm}$, were treated by shifting step by step transversely to the electron beam. The radiation monitoring system allowed obtaining three-dimensional dose distribution in experimental samples so as to make the radiationchemical experiments more precise. factor for given chemical content of the material is the dose of electron/bremsstrahlung radiation. The type of radiation and the dose rate also have effect and should be considered when choosing the conditions of the radiationinduced polymerization of materials. Two-sided irradiation of thick PMW samples or sample stacks, which thickness is more than the electron range, was used. For thick high-density items it is useful to convert the electron beam to bremsstrahlung which has higher penetration ability and therefore produces more homogeneous absorbed dose.

The processes of polymerization and co-polymerization of monomer in capillary-porous or dispersed filler differs from one in pure monomer binder and has catalytic nature. That is why dispersion or specific surface of the base material have as much influence on polymerization degree as concentration of sensitizing impurities or temperature.

Methods of optimizing parameters of radiation treatment targeted to maximize productivity of the accelerator and obtain the most homogeneous dose distribution in the volume of processed materials were developed. On the basis of our experimental data as well as computer simulation of the radiation field in the target material a mathematical model of the polymerization process was built. The parameters of the model are electron energy, beam current, exposure time, temperature, geometric parameters of the scanning beam and the container, wood density, the chemical formula of the binder and impregnation degree. There are variants of the model considering two-sided irradiation of the container and conversion electron beam to bremsstrahlung. For the particular base-binder pair unknown parameters of radiation polymerization process are determined by a few technological experiments, and then one can calculate the optimal conditions of the process in terms of productivity and homogeneity of dose. In general the dose 5-50 Mrad is necessary to achieve the desired degree of polymerization, and it takes 5-30 minutes of irradiation on such accelerator as we used. Overall productivity of our pilot model of installation is $20000-40000 \mathrm{~m}^{3}$ of parquet flooring per year.

Pilot series of goods and experimental examples of polymer-modified wood were manufactured. The following characteristics of new PMW were measured: mass density; hardness; bursting and bearing strength, toughness, compression and elasticity modules, abrasive wear, thermal expansion coefficient, moisture resistance; shape stability; waterproof, thermoinsulation and dielectric characteristics; fire resistance; biological and chemical resistance. It was shown that because of modification the wood the hardness increases 5-10 times, elasticity modulus increases 2-5 times, abrasion wear decreases 2-4 times, water absorption decreases 20-50 
material to desired color, for example make the inexpensive wood looking like ebony or mahogany.

Some characteristics of modified with methylmethacrylat wood are compared with that all mechanical and operational properties of modified soft wood are better than properties of natural oak.

Table 1. Comparative characteristics of natural and modified with methylmethacrylat wood

\begin{tabular}{|l|c|l|l|l|l|}
\hline Characteristic & \multicolumn{2}{|c|}{ birch } & \multicolumn{2}{c|}{ aspen } & oak \\
\hline & original & modified & original & modified & original \\
\hline Density, g/cub.sm & 0.63 & $0.9-1.2$ & 0.47 & $0.9-1.2$ & 0.69 \\
\hline Hardness, MPa & $40-48$ & $120-180$ & $25-30$ & $110-160$ & $65-70$ \\
\hline Cross-breaking strength,MPa & $80-90$ & $200-260$ & $70-80$ & $160-200$ & $90-110$ \\
\hline Compressive strength, MPa & $45-55$ & $100-130$ & $30-40$ & $100-120$ & $45-58$ \\
\hline Impact strength, KJ/sq.m & $70-80$ & $120-260$ & $70-80$ & $120-220$ & $65-80$ \\
\hline Abrasion wear, mm & $0.5-0.6$ & 0.2 & 1.2 & 0.35 & 0.5 \\
\hline Water absorption for 24 hrs, $\%$ & $70-80$ & $3-6$ & $70-95$ & $3-6$ & $25-30$ \\
& & & & & \\
\hline
\end{tabular}

\section{CONCLUSION}

An advantage of the developed technology is the possibility to utilize wastes of woodworking industry such as wood, chipboard and veneer cuttings, sawdust and chips. Such soft low-grade wood as poplar, aspen, alder, and birch make high quality materials when modified with synthetic polymer. Polymer modified materials may be used for manufacturing such top-quality goods as furniture, window frames, doors, parquet, wall siding, gear drives, etc.

High hardness and shape stability, high thermoinsulation and waterproof characteristics, chemical and biological durability make the materials ideal for use in heavy conditions such as chemically aggressive environment or wet tropical climate, another prospective area of application is preservation and restoration of antiquities.

\section{REFERENCES}

[1] I. Zalubovskiy, A. Avilov, G. Popov, V. Rudychev , Proc. Int. Conf." Physics in Ukraine", Kiev, Ukraine, June, p.234-236, (1993).

[2] I. Zalubovskiy, A. Avilov, G. Popov, V. Rudychev, Bulletin of the American Physical Society 40, N3. p.1098, (1995).

[3] G. Popov, I. Zalubovskiy, A. Avilov, V. Rudychev, Bulletin of the American Physical Society 42, N3. p.1375, (1997).

[4]. G. F. Popov, V. A. Deryuga. ECAART'97, Eindhoven, the Netherlands, p.176, (1977).

[5]. G.Popov, A.Avilov, V.Rudychev, I.Zalyubovsky, "Internàtional

Technology Transfer from the Newly Independent States of the Former Soviet Union", ITT-98, Des Moines, Iowa, USA, (1998).

[6]. I.Zalyubovsky, A.Avilov, G.Popov, V.Rudychev Proc.Int. Conf. "Cyclotrons and Applications", 15-19 March 1997, Cairo, Egypt, p. 281-288, (1998).

[7].I.Zalyubovsky, A.Kalinichenco, Yu.Kresnin, G.Popov Proc.Int. Conf. "Cyclotrons and Applications", 15-19 March 1997, Cairo, Egypt , p.434-443, (1998).

[8] A. Kalinichenko, G. Popov, Proc. Intern. IEEE Nuclear Scince Symposium. Anaheim, USA, Nov. 3-9, p. 83, (1996).

[9] G. Popov, V. Deryuga, A. Kalinichenko, Yu. Kresnin. Proc. Intern. Conf. on Accelerator and Large Experimental Physics Control Systems, Chicago, USA, Oct.30, p.954-956, (1995). 